\title{
Association Between Serum Magnesium Level and Metabolic Syndrome Prevalence in Chinese Adults: A Population Study
}

\author{
Mengyao Zhou, Jing Wang, Junhao Wang, Wenjie Yang* \\ Precision Nutrition Innovation Institute, School of Public Health, Zhengzhou University, Zhengzhou, China
}

Email address:

ywjie@zzu.edu.cn (Wenjie Yang)

${ }^{*}$ Corresponding author

\section{To cite this article:}

Mengyao Zhou, Jing Wang, Junhao Wang, Wenjie Yang. Association Between Serum Magnesium Level and Metabolic Syndrome Prevalence in Chinese Adults: A Population Study. American Journal of Internal Medicine. Vol. 9, No. 4, 2021, pp. 198-203.

doi: $10.11648 /$ j.ajim.20210904.16

Received: July 18, 2021; Accepted: July 28, 2021; Published: August 4, 2021

\begin{abstract}
Aims: To assess the relationship between the level of serum magnesium and metabolic syndrome in Chinese adults. Methods: We used data from the China Health and Nutrition Survey (CHNS) conducted in 2009, and conducted investigated a cross-sectional study of 7364 Chinese subjects. A total of 1676 people (659 men and 1067 women) met the criteria of metabolic syndrome. Rank correlation analysis and binary Logistic regression analysis were performed to analyze the influence of serum magnesium concentrations, provinces and gender on the prevalence of metabolic syndrome. Results: The prevalence of metabolic syndrome is $22.8 \%$. The concentration of serum magnesium in people with metabolic syndrome is higher than that in healthy groups $(0.95 \mathrm{mmol} / \mathrm{L}$ vs $0.93 \mathrm{mmol} / \mathrm{L}, P<0.001)$. The serum magnesium concentration and the prevalence of metabolic syndrome are higher in magnesium-rich areas than in non-magnesium-rich ones, with significant differences. (serum magnesium: $\mathrm{t}=7.078, P<0$. 001; metabolic syndrome: $\chi^{2}=46.529, P<0$. 001.) The Spearman rank correlation coefficient between serum magnesium level and the prevalence of metabolic syndrome is 0.903 in males, and 0.891 in females. All participants were divided into four groups according to the serum magnesium concentration quartile. The risk of metabolic syndrome increases by 1.595 times (95\% CI 1.358-1.873). Conclusion: Higher serum magnesium concentration may be a risk factor for metabolic syndrome. Serum magnesium concentration is moderately and even highly correlated with the prevalence of metabolic syndrome, and there is a gender difference in this relation.
\end{abstract}

Keywords: Metabolic Syndrome, Serum Magnesium, Cross-Sectional Study, Adults

\section{Introduction}

Metabolic syndrome is now widely considered as a group of metabolic abnormalities, including central obesity, hypertension, glucose intolerance, and dyslipidemia (hypertriglyceridemia and low HDL cholesterol levels) [1]. To date, the metabolic syndrome has reached pandemic level worldwide. The situation in China is not optimistic, because a recent study claimed that the average prevalence of metabolic syndrome in 31 provinces of China in 2010 was $33.9 \%$ (31.0\% for men and $36.8 \%$ for women) [2]. The metabolic syndrome has posed increasing risk for diabetes and cardiovascular diseases (CVD) [3]. Therefore, it is important to understand the risk factors of metabolic syndrome and prevent metabolic syndrome to public health.

It is widely believed that dietary and lifestyle modifications are critical to reducing the incidence of metabolic syndrome. Magnesium is usually found in whole grains, green leafy vegetables, legumes and nuts [4]. And magnesium, an essential nutrient, as the second intracellular cation [5], is the key cofactor for hundreds of enzymatic reactions, including protein and nucleic acid synthesis, energy metabolism, and insulin secretion and action [6,7]. Although the role of magnesium is not fully understood, its significance is unquestionable, especially in chronic diseases, and the development of metabolic syndrome [8]. 
Many studies have investigated the relationship between serum magnesium and the prevalence of metabolic syndrome, but the results are inconsistent. Some suggested that serum magnesium levels were significantly lower in metabolic syndrome groups compared with healthy controls [9-11], Among the components of metabolic syndrome, dyslipidemia and hypertension were related to low serum magnesium levels [9]. And Ghasemi found that there was a significant negative correlation between blood glucose and serum magnesium [11]. Whereas others found no association or even positive association [12-14]. To find out its cause, we speculate that this might be due to differences in selected populations.

Furthermore, few have examined their correlation between serum magnesium level and metabolic syndrome in China. Therefore, we aim to investigate the association between serum magnesium level and the prevalence of metabolic syndrome, using the China health and nutrition survey (CHNS) database in 2009.

\section{Methods}

\subsection{Data Collection and Sources}

The CHNS is a household-based longitudinal cohort study. From 1989 to 2015,10 survey rounds were conducted in 9 provinces across China, including Liaoning, Heilongjiang, Hunan, Shandong, Hubei, Henan, Jiangsu, Guangxi, Guizhou. Multistage, random clustering method was used to extract the samples from each province. Counties in the nine provinces were stratified according to income (low, middle, and high) and four counties were randomly selected in each province by using the weighted sampling scheme. Data for the present study was obtained from 2009. There were 8708 eligible participants 18 years or older. 1236 participants who did not have Mets components information were excluded from the analysis. We excluded 30 participants due to missing data on serum magnesium or outliers (outliers were defined as values standing outside of 3 times the interquartile range). The final analysis included 7364 participants who gave written informed consent for their participation in the survey. The research was approved by the institutional review committee of the University of North Carolina at Chapel Hill and the Chinese Center for Disease Control and Prevention.

\subsection{Measures}

Blood collection, processing, and storage followed the same standard operating procedures. Serum magnesium levels in millimoles per liter ( $\mathrm{mmol} / \mathrm{L})$ were measured using the Xylidyl Blue colorimetric method. The plasma lipid and lipoprotein, including total cholesterol (TC), triglyceride (TG), HDL-C, LDL-C, were determined by the enzyme-colorimetric method. Fasting plasma glucose concentrations were enzymatically measured [15]. The questionnaire collected data on age, sex, smoking, alcohol consumption, education attainment, geographical area (north/south), residence area (urban/rural), smoking habits, alcohol and tea intake. Anthropometric measurements were performed by well-trained staff members.

Waist circumference (WC, cm) was measured in $0.1 \mathrm{~cm}$ unit at the midpoint between the lowest rib and the iliac crest in a standing position. Body mass index (BMI) was calculated based on the measured values of weight and height [BMI = weight $(\mathrm{kg}) /$ height $\left.(\mathrm{m})^{2}\right]$ [16].

\subsection{Definition of the Metabolic Syndrome}

Metabolic syndrome (Mets) was diagnosed by the International Diabetes Federation (IDF) consensus worldwide definition of the Mets (International Diabetes Federation, 2005) [17]. In this definition, central obesity is considered an essential diagnostic element for Mets, and be defined as waist circumference (Chinese-specific values: $\mathrm{WC} \geq 90 \mathrm{~cm}$ in men or $\mathrm{WC} \geq 80 \mathrm{~cm}$ in women), According to the new definition, for a person to be defined as having the Mets, they must have central obesity plus any two of four additional factors. These four factors are: 1 . Hypertriglyceridemia: TG $>1.7 \mathrm{mmol} / \mathrm{L}(>150 \mathrm{mg} / \mathrm{dl}) .2$. Low HDL cholesterol: HDL-C $<1.03 \mathrm{mmol} / \mathrm{L}$ in men or 1.3 $\mathrm{mmol} / \mathrm{L}$ in women $(<40 \mathrm{mg} / \mathrm{dL}$ for $\mathrm{men},<50 \mathrm{mg} / \mathrm{dL}$ for women). 3. High blood pressure: Blood pressure $\geq 130$ $\mathrm{mmHg}$ SBP or $85 \mathrm{mmHg}$ DBP or treatment of previously diagnosed hypertension. 4. High fasting glucose: Fasting glucose $\geq 5.6 \mathrm{mmol} / \mathrm{L}(\geq 100 \mathrm{mg} / \mathrm{dL})$ or previously diagnosed Type 2 diabetes.

\subsection{Statistical Analysis}

Baseline characteristics of study participants are described as means and standard deviations (SD) or median for quantitative variables, and percentages (numbers) for categorical variables. Between-group differences in quantitative variables were assessed with the t-test (for a normal distribution) or the Mann-Whitney test (for skewed variables). The chi-square test was used to compare difference groups of categorical variables. Serum magnesium was grouped by decile, and the relationship between serum magnesium and Mets prevalence was investigated using the Spearman rank correction test. The strength of correlation was determined based on Spearman rank correlation coefficient $(r)$ values: weak $(r<0.7)$, moderate $(0.7 \leq r<0.9)$, strong $(r \geq 0.9)$.

All the participants were divided into four groups according to the quartile of serum magnesium and the quartiles was used to compare components and other lifestyle factors. Logistic regression was used to calculate odds ratios (ORs) with $95 \%$ CIs for the association between serum magnesium and Mets risk. We calculated multivariate-adjusted ORs by adjusting for potential risk factors of Mets, (including age, sex, geographical area, residential area, smoking status, alcohol drinking, tea drinking et al).

Statistical analyses were performed using SPSS software (version 24.0), P-value of $<0.05$ was considered statistically significant. 


\section{Results}

\subsection{Characteristics of the Participants}

Table 1 shows the characteristics of the participants according to metabolic syndrome status. Questionnaires were administered to 7364 subjects in total, including 3435 (46.6\%) males and 3929 (53.4\%) females. 1676 (22.8\%) patients met the defined criteria for metabolic syndrome.
Female subjects have a significantly higher prevalence of metabolic syndrome than the male subjects $(26.7 \%$ vs $18.2 \%)$. Subjects with Mets are older, more likely to live in urban areas, less well-educated and more frequent tea drinkers, higher in BMI, or larger in waist circumference. In the case of smoking and alcohol consumption, however, the proportions of No Mets group are even higher, which might be a result of gender difference.

Table 1. Characteristics of the study population by Mets status.

\begin{tabular}{llll}
\hline & Mets & No Mets & p-value \\
\hline $\mathrm{N}$ & 1676 & 5688 & $<0.001$ \\
Age (years) & $56.25 \pm 12.95$ & $49.27 \pm 15.53$ & $<0.001$ \\
Females (\%) & 62.7 & 50.6 & 0.017 \\
Urban (\%) & 34.7 & 31.6 & $<0.001$ \\
Education (high) (\%) & 7.9 & 11.5 & $<0.001$ \\
Current smoker (\%) & 24.8 & 32.7 & $<0.001$ \\
Current alcohol drinker (\%) & 27.4 & 36.5 & 0.875 \\
Tea drinker (\%) & 36.5 & 37.2 & $<0.001$ \\
BMI & $26.39 \pm 3.26$ & $22.48 \pm 3.02$ & $<0.001$ \\
Waist circumference (cm) & $92.84 \pm 7.74$ & $79.92 \pm 9.23$ & $<0.001$ \\
SBP (mmHg) & $136.57 \pm 20.05$ & $121.49 \pm 17.37$ & $<0.001$ \\
DBP (mmHg) & $86.37 \pm 11.01$ & $78.40 \pm 10.55$ & $<0.001$ \\
TC (mmol/L) & $5.21 \pm 1.04$ & $4.79 \pm 0.97$ & $<0.001$ \\
HDL-C (mmol/L) & $1.23 \pm 0.43$ & $1.50 \pm 0.45$ & $<0.001$ \\
LDL-C (mmol/L) & $3.17 \pm 1.09$ & $2.96 \pm 0.95$ & $<0.001$ \\
TG (mmol/L) & $2.64 \pm 1.87$ & $1.35 \pm 1.01$ & $<0.001$ \\
Glucose (mmol/L) & $6.28 \pm 2.09$ & $5.18 \pm 1.10$ & \\
\hline
\end{tabular}

\subsection{Association Between Serum Magnesium and Mets}

Table 2 presents the relationship between serum magnesium levels and metabolic syndrome in Chinese adults. Compared to those No Mets group, serum magnesium levels are significantly higher in subjects with metabolic syndrome $(0.95 \mathrm{mmol} / \mathrm{L}$ vs $0.93 \mathrm{mmol} / \mathrm{L}, \quad \mathrm{P}<0.001)$. According to the geographic distribution of Chinese magnesium mines, all provinces can be divided into magnesium-rich provinces and non-magnesium-rich provinces. Among these provinces, magnesium-rich provinces were Liaoning and Shandong, other provinces were non-magnesium-rich areas. The mean serum magnesium level of subjects was $0.95 \mathrm{mmol} / \mathrm{L}$ in Mets group, and No Mets group was $0.93 \mathrm{mmol} / \mathrm{L}$. Furthermore, magnesium-rich provinces show a significantly higher prevalence of Mets than non-magnesium-rich provinces.

Table 2. The relationship between serum magnesium content and Mets in Chinese adults.

\begin{tabular}{llll}
\hline & Mets & No Mets & p-value \\
\hline $\mathrm{N}$ & $1676(22.8)$ & $5688(77.2)$ & \\
magnesium-rich areas & $503(28.7)$ & $1248(71.3)$ & \\
non-magnesium-rich areas & $1173(20.9)$ & $4440(79.1)$ & $\chi^{2}=46.529$ \\
Serum magnesium (mmol/) & $0.95 \pm 0.09$ & $0.93 \pm 0.09$ & $\mathrm{t}=7.078$ \\
\hline
\end{tabular}

\subsection{Correlation Analysis of Serum Magnesium Level with Prevalence of Mets}

We classified study subjects into ten groups in terms of serum magnesium level deciles as mentioned above. The average concentrations in these groups are $0.77 \mathrm{mmol} / \mathrm{L}$, $0.85 \mathrm{mmol} / \mathrm{L}, 0.89 \mathrm{mmol} / \mathrm{L}, 0.92 \mathrm{mmol} / \mathrm{L}, 0.93 \mathrm{mmol} / \mathrm{L}$, $0.95 \mathrm{mmol} / \mathrm{L}, 0.98 \mathrm{mmol} / \mathrm{L}, 1.00 \mathrm{mmol} / \mathrm{L}, 1.03 \mathrm{mmol} / \mathrm{L}$, $1.10 \mathrm{mmol} / \mathrm{L}$, respectively. As shown in Figure 1, the prevalence of Mets gradually and markedly increases with increasing serum magnesium deciles in both male and female groups. Spearman's rank correlation analysis was performed using the serum magnesium level and prevalence of Mets in each group. Spearman rank correlation coefficient was employed to assess correlations. The results show that the Spearman's rank correlation coefficient between magnesium level and prevalence of Mets is 0.903 in male group $(\mathrm{P}<0.001)$. And Spearman rank correlation coefficient between magnesium level and prevalence of Mets is 0.891 female group $(\mathrm{P}<0.001)$. 


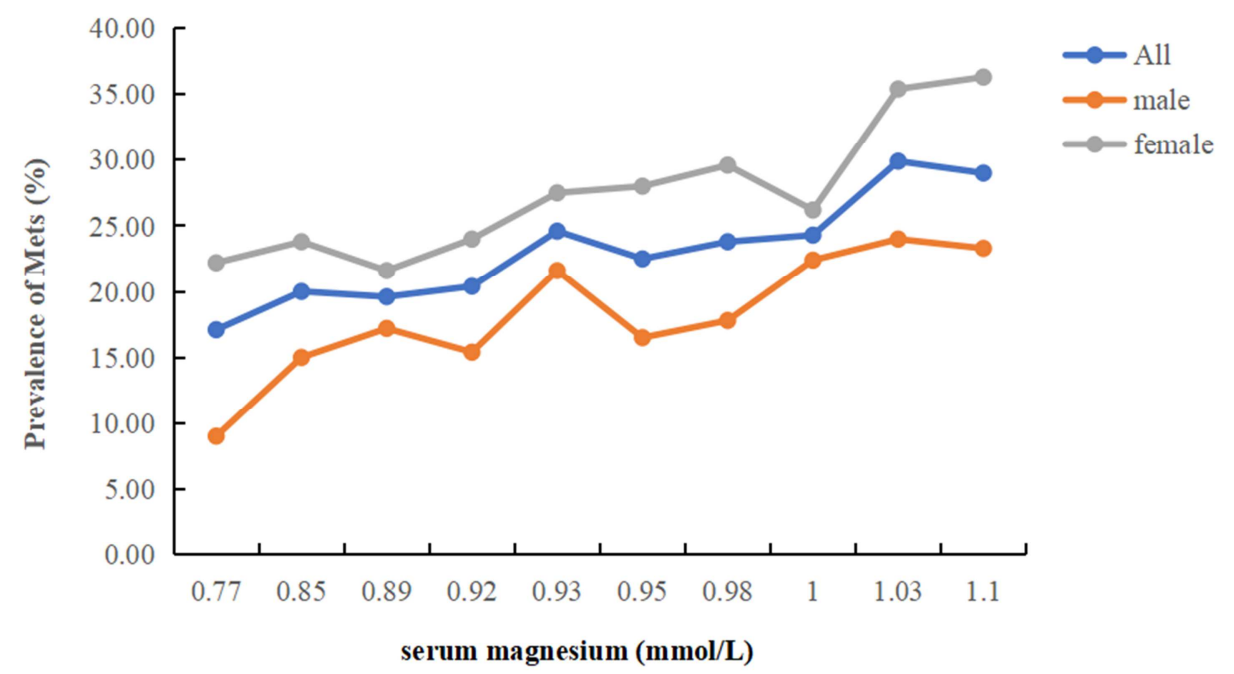

Figure 1. Spearman rank correlation analysis of Serum Magnesium level with prevalence of Mets.

\subsection{Multiple Logistic Regression Analysis of Mets Prevalence}

Because the magnitude of serum magnesium level deciles was relatively small. We followed the grouping methods of previous literature [18] and divided the participants into four groups in terms of serum magnesium level according to the quartiles of distribution. The average concentrations in these groups are $0.83 \mathrm{mmol} / \mathrm{L}, 0.91 \mathrm{mmol} / \mathrm{L}, 0.96 \mathrm{mmol} / \mathrm{L}, 1.05$ $\mathrm{mmol} / \mathrm{L}$, respectively.

We conducted a multiple logistic regression analysis, using dichotomous variable Mets and Mets components as the dependent variable, sex, age, province and serum magnesium as the independent variable, smoking, alcohol drinking, and tea drinking as covariates for model 2. Among these variables, serum magnesium content was rank variable, sex and province were binary variables.

The results are presented in Table 3. The crude OR for Mets comparing the highest to the lowest quartile of serum magnesium is $1.714(95 \% \mathrm{Cl} 1.468-2.002)$. After multivariable adjustment, this association is still statistically significant $(\mathrm{OR}=1.595,95 \% \mathrm{Cl} 1.358-1.873)$. In the logistic regression analysis, statistically significant associations of triglycerides, blood glucose or waist perimeter with serum magnesium are observed between the lowest quartile and highest quartile. The older the age is, the higher the risk of Mets is $(\mathrm{OR}=1.031$, $95 \% \mathrm{Cl} 1.027-1.036)$. Meanwhile, more women than men have metabolic syndrome $(\mathrm{OR}=1.771,95 \% \mathrm{Cl} 1.542-2.035)$. Compared with the non-magnesium-rich provinces, the risk of Mets in magnesium-rich provinces is significantly higher $(\mathrm{OR}=1.496,95 \% \mathrm{Cl} 1.318-1.697)$.

Table 3. Association between serum magnesium and Mets in the study population.

\begin{tabular}{|c|c|c|c|c|}
\hline & Q1 & Q2 & Q3 & Q4 \\
\hline Magnesium $(\mathrm{mmol} / \mathrm{L})$ & 0.8255 & 0.9112 & 0.9642 & 1.0470 \\
\hline MS & 1.00 (reference) & $1.159(0.984-1.366)$ & $1.282(1.097-1.499)$ & $1.714(1.468-2.002)$ \\
\hline Model 1 & & $1.159(0.980-1.370)$ & $1.241(1.058-1.456)$ & $1.667(1.422-1.956)$ \\
\hline Model 2 & & $1.114(0.942-1.318)$ & $1.194(1.017-1.403)$ & $1.595(1.358-1.873)$ \\
\hline Waist perimeter & 1.00 (reference) & $1.052(0.921-1.202)$ & $1.168(1.029-1.327)$ & $1.369(1.201-1.560)$ \\
\hline Model 1 & & $1.069(0.930-1.228)$ & $1.202(1.052-1.375)$ & $1.471(1.281-1.689)$ \\
\hline Model 2 & & $1.000(0.869-1.151)$ & $1.134(0.991-1.299)$ & $1.379(1.199-1.587)$ \\
\hline Model 1 & & $1.148(0.991-1.329)$ & $1.301(1.131-1.497)$ & $1.788(1.553-2.059)$ \\
\hline Model 2 & & $1.131(0.976-1.311)$ & $1.283(1.115-1.476)$ & $1.760(1.527-2.028)$ \\
\hline HDL & 1.00 (reference) & $1.053(0.904-1.227)$ & $1.072(0.925-1.241)$ & $0.941(0.806-1.097)$ \\
\hline Model 1 & & $1.077(0.923-1.258)$ & $1.131(0.974-1.314)$ & $1.028(0.879-1.204)$ \\
\hline Model 2 & & $1.075(0.920-1.256)$ & $1.135(0.976-1.319)$ & $1.037(0.885-1.214)$ \\
\hline Hypertension & 1.00 (reference) & $1.047(0.916-1.196)$ & $1.200(1.056-1.364)$ & $1.329(1.165-1.515)$ \\
\hline Model 1 & & $1.018(0.879-1.179)$ & $1.069(0.928-1.231)$ & $1.110(0.961-1.283)$ \\
\hline Glucose & 1.00 (reference) & $1.074(0.922-1.250)$ & $1.221(1.057-1.411)$ & $1.575(1.362-1.820)$ \\
\hline Model 1 & & $1.064(0.910-1.243)$ & $1.145(0.987-1.328)$ & $1.435(1.236-1.665)$ \\
\hline Model 2 & & $1.045(0.893-1.222)$ & $1.116(0.962-1.296)$ & $1.388(1.195-1.613)$ \\
\hline
\end{tabular}

Notes: Values are odds ratio ORs (95\% confidence interval CI). Model 1 is adjusted for age (continuous), sex (males or females). Model 2 is adjusted for age (continuous), sex (males or females), geographic region (magnesium-rich or non-magnesium-rich), current smoker (yes/no), current alcohol drinker (yes/no), tea drinker (yes/no). 


\section{Discussion}

As an essential element, magnesium in the human body is mostly located in the bone tissues [19]. Magnesium serves a variety of important biological functions [20]. It is a cofactor by over 300 enzymes in biological processes [7, 21], include, but are not limited to, protein synthesis, energy generation and storage, reproduction, DNA replication, RNA transcription, and mitochondrial membrane stabilization [22, 23]. And it is important for the transmission of nerve impulses and muscle contraction [24]. In serum, it plays pivotal roles in blood pressure, and glucose and insulin metabolism [25-27]. Magnesium plays an important role in disease prevention and overall health due to its many functions in the body. However, the association between serum magnesium and Mets prevalence is still unclear. Our study was solely based on the 2009 CHNS data, in which we aimed to investigate the association between Mets and serum magnesium concentration. The findings may provide a new scientific and theoretical basis for the prevention and control of Mets.

Our study has shown that serum magnesium level is higher in patients with Mets than in control group, the difference being statistically significant. Compared with the non-magnesium-rich provinces, participants in magnesium-rich provinces have significantly higher serum magnesium level and prevalence of Mets. With increasing serum magnesium level, the prevalence of Mets rises gradually in both male and female groups. Therefore, magnesium may be a risk factor for metabolic syndrome. Spearman's rank-correlation test was used to evaluate the correlation between these two variables to further understand this finding. We find that magnesium level is highly correlated with Mets in male group with a correlation coefficient of 0.903 . And the correlation coefficient is 0.891 for female group, indicating a moderate correlation between Mets prevalence and magnesium level. In the multiple logistic regression analysis, the risk of Mets increases by 1.595 times for every one grade decrease in serum magnesium level. Thus, these evidences suggest that serum magnesium level can contribute to Mets. Results of prior studies have, however, been inconsistent. Maria et al. found that serum magnesium level of Mets participants was lower than that of normal people [28]. Our findings agree with one study [13]. Although some studies did not find a significant difference [29], we speculate that this might be due to differences in study designs, selected populations and/or the ratio of biosynthetic enzymes and metabolism enzymes.

Admittedly, certain limitations exist in our study: 1) As cross-sectional study, we could not establish the causality between metabolic syndrome and serum magnesium. 2) Although we used the updated definition of metabolic syndrome, currently there is no standard definition for Mets in the Asian population. To the best of our knowledge, this is the first study to comprehensively analyze the association between Mets and serum magnesium, which includes more than 7000 participants from nine provinces in China. In the future, additional prospective studies should be taken to reveal the effect and mechanism of magnesium in metabolic diseases.

\section{Conclusion}

Overall, our research shows that magnesium-rich provinces show a significantly higher prevalence of Mets than non-magnesium-rich provinces. And serum magnesium levels are significantly higher in subjects with metabolic syndrome compared to those without metabolic syndrome. Taken together, the increase of serum magnesium level is a risk factor for metabolic syndrome. The concentrations of serum magnesium are highly or moderately correlated with Mets, depending on gender. And our results also indicate increasing serum magnesium level to be related glucose and lipid metabolism disorders.

\section{References}

[1] Evaluation Expert Panel on Detection, Adults Treatment of High Blood Cholesterol in. Executive Summary of The Third Report of The National Cholesterol Education Program (NCEP) Expert Panel on Detection, Evaluation, And Treatment of High Blood Cholesterol In Adults (Adult Treatment Panel III) [J]. JAMA, 2001, 285 (19): 2486-2497.

[2] E. S. Ford. Risks for all-cause mortality, cardiovascular disease, and diabetes associated with the metabolic syndrome: a summary of the evidence [J]. Diabetes Care, 2005, 28 (7): 1769-1778.

[3] Z. Ren, A. Zhao, Y. Wang, et al. Association between Dietary Inflammatory Index, C-Reactive Protein and Metabolic Syndrome: A Cross-Sectional Study [J]. Nutrients, 2018, 10 (7).

[4] N. E. Saris, E. Mervaala, H. Karppanen, et al. Magnesium. An update on physiological, clinical and analytical aspects [J]. Clin Chim Acta, 2000, 294 (1-2): 1-26.

[5] G. Piuri, M. Zocchi, M. Della Porta, et al. Magnesium in Obesity, Metabolic Syndrome, and Type 2 Diabetes [J]. Nutrients, 2021, 13 (2).

[6] M. Barbagallo, L. J. Dominguez, A. Galioto, et al. Role of magnesium in insulin action, diabetes and cardio-metabolic syndrome X [J]. Mol Aspects Med, 2003, 24 (1-3): 39-52.

[7] R. J. Elin. Magnesium metabolism in health and disease [J]. Dis Mon, 1988, 34 (4): 161-218.

[8] H. Khosravi-Boroujeni, N. Sarrafzadegan, N. Mohammadifard, et al. Consumption of sugar-sweetened beverages in relation to the metabolic syndrome among Iranian adults $[\mathrm{J}]$. Obes Facts, 2012, 5 (4): 527-537.

[9] F. Guerrero-Romero, M. Rodriguez-Moran. Low serum magnesium levels and metabolic syndrome [J]. Acta Diabetol, 2002, 39 (4): 209-213.

[10] S. L. Volpe. Magnesium, the metabolic syndrome, insulin resistance, and type 2 diabetes mellitus [J]. Crit Rev Food Sci Nutr, 2008, 48 (3): 293-300. 
[11] A. Ghasemi, S. Zahediasl, L. Syedmoradi, et al. Low serum magnesium levels in elderly subjects with metabolic syndrome [J]. Biol Trace Elem Res, 2010, 136 (1): 18-25.

[12] S. H. Park, S. K. Kim, Y. J. Bae. Relationship between serum calcium and magnesium concentrations and metabolic syndrome diagnostic components in middle-aged Korean men [J]. Biol Trace Elem Res, 2012, 146 (1): 35-41.

[13] Y. Yu, Z. Cai, J. Zheng, et al. Serum levels of polyunsaturated fatty acids are low in Chinese men with metabolic syndrome, whereas serum levels of saturated fatty acids, zinc, and magnesium are high [J]. Nutr Res, 2012, 32 (2): 71-77.

[14] Y. J. Bae, M. K. Choi. Magnesium intake and its relevance with antioxidant capacity in Korean adults [J]. Biol Trace Elem Res, 2011, 143 (1): 213-225.

[15] B. Xi, D. He, Y. Hu, et al. Prevalence of metabolic syndrome and its influencing factors among the Chinese adults: the China Health and Nutrition Survey in 2009 [J]. Prev Med, 2013, 57 (6): 867-871.

[16] P. Song, X. Li, D. Gasevic, et al. BMI, Waist Circumference Reference Values for Chinese School-Aged Children and Adolescents [J]. Int J Environ Res Public Health, 2016, 13 (6).

[17] K. G. Alberti, P. Zimmet, J. Shaw. Metabolic syndrome--a new world-wide definition. A Consensus Statement from the International Diabetes Federation [J]. Diabet Med, 2006, 23 (5) 469-480.

[18] J. Zhang, H. Wang, Z. Wang, et al. Association between Toenail Magnesium and Type 2 Diabetes in Chinese Adults [J]. Nutrients, 2017, 9 (8).

[19] U. Grober, J. Schmidt, K. Kisters. Magnesium in Prevention and Therapy [J]. Nutrients, 2015, 7 (9): 8199-8226.

[20] Y. Zhang, P. Xun, R. Wang, et al. Can Magnesium Enhance Exercise Performance? [J]. Nutrients, 2017, 9 (9).
[21] N. Veronese, S. Watutantrige-Fernando, C. Luchini, et al Effect of magnesium supplementation on glucose metabolism in people with or at risk of diabetes: a systematic review and meta-analysis of double-blind randomized controlled trials [J]. Eur J Clin Nutr, 2016, 70 (12): 1354-1359.

[22] C. H. Bohl, S. L. Volpe. Magnesium and exercise [J]. Crit Rev Food Sci Nutr, 2002, 42 (6): 533-563.

[23] V. Chubanov, T. Gudermann, K. P. Schlingmann. Essential role for TRPM6 in epithelial magnesium transport and body magnesium homeostasis [J]. Pflugers Arch, 2005, 451 (1): 228-234.

[24] E. Wesselink, R. M. Winkels, H. van Baar, et al. Dietary Intake of Magnesium or Calcium and Chemotherapy-Induced Peripheral Neuropathy in Colorectal Cancer Patients [J]. Nutrients, 2018, 10 (4).

[25] G. Paolisso, M. Barbagallo. Hypertension, diabetes mellitus, and insulin resistance: the role of intracellular magnesium [J]. Am J Hypertens, 1997, 10 (3): 346-355.

[26] J. B. Morais, J. S. Severo, L. R. Santos, et al. Role of Magnesium in Oxidative Stress in Individuals with Obesity [J]. Biol Trace Elem Res, 2017, 176 (1): 20-26.

[27] M. Barbagallo, L. J. Dominguez. Magnesium metabolism in type 2 diabetes mellitus, metabolic syndrome and insulin resistance [J]. Arch Biochem Biophys, 2007, 458 (1): 40-47.

[28] L. Lima Mde, T. Cruz, L. E. Rodrigues, et al. Serum and intracellular magnesium deficiency in patients with metabolic syndrome--evidences for its relation to insulin resistance [J]. Diabetes Res Clin Pract, 2009, 83 (2): 257-262.

[29] D. Simmons, S. Joshi, J. Shaw. Hypomagnesaemia is associated with diabetes: Not pre-diabetes, obesity or the metabolic syndrome [J]. Diabetes Res Clin Pract, 2010, 87 (2): 261-266. 